\title{
Effect of the integration scheme on the rotation of non-spherical particles with the Discrete Element Method.
}

\author{
Joaquín Irazábal · Fernando Salazar · Miquel Santasusana · Eugenio \\ Oñate
}

Received: date / Accepted: date

\begin{abstract}
The Discrete Element Method (DEM) is an emerging tool for the calculation of the behaviour of bulk materials. One of the key features of this method is the explicit integration of the motion equations. Explicit methods are rapid, at the cost of a limited time step to achieve numerical stability. First or second order integration schemes based on a Taylor series are frequently used in this framework and showed to be accurate for the translational and rotational motion of spherical particles. However, they may lead to relevant inaccuracies when non-spherical particles are used since the orientation implies a modification in the second order inertia tensor in the inertial reference frame. Specific integration schemes for non-spherical particles have been proposed in the literature, such as the $4^{\text {th }}$ order Runge-Kutta scheme presented by Munjiza et al. and the predictor-corrector scheme developed by Zhao and van Wachem which applies the direct multiplication algorithm for integrating the orientation. In this work, both methods are adapted to be used together with a Velocity Verlet scheme for the translational in-
\end{abstract}

\section{J. Irazábal}

International Centre for Numerical Methods in Engineering (CIMNE), Barcelona, Spain

E-mail: jirazabal@cimne.upc.edu

F. Salazar

International Centre for Numerical Methods in Engineering (CIMNE), Barcelona, Spain

\section{Santasusana}

International Centre for Numerical Methods in Engineering (CIMNE), Barcelona, Spain

\section{E. Oñate}

International Centre for Numerical Methods in Engineering (CIMNE), Barcelona, Spain

Polytechnic University of Catalonia, Barcelona, Spain tegration. The performance of the resulting schemes, as well as that of the direct integration method, is assessed, both in benchmark tests with analytical solution and in real-scale problems. The results suggest that the the $4^{t h}$ order Runge-Kutta and the Zhao and van Wachem schemes are clearly more accurate than the direct integration method without increasing the computational time.

Keywords Discrete element method · granular material · non-spherical particles · clusters of spheres . explicit rotational integration

\section{Introduction}

Granular materials are widely used in large civil constructions such as dams, embankments and railways [45]. Also, bulk materials are treated, processed or transported in many industrial processes [40]. In both cases, a better knowledge of the mechanical behaviour of this kind of materials is essential to improve the design of infrastructures and to increase the efficiency of industrial processes.

Traditionally, experimental tests were the only option to evaluate the behaviour of these materials, by means of laboratory tests in specific devices. Nowadays, the development of numerical models allows combining experimental and numerical approaches to face these problems, with reduction in overall cost and increase in efficiency.

Several numerical approaches from continuum mechanics have been adapted to model granular materials, such as the Material Point Method (MPM) [19] or the Particle Finite Element Method (PFEM) [30, 31, 36], among others $[11,9,27,6]$. The Discrete Element Method (DEM) was specifically designed by Cundall 
and Strack [8] to simulate bulk materials in a natural way. In the DEM, each material grain is considered as a rigid body whose behaviour is governed by contact interactions with other particles and boundaries. After evaluating the forces over each Discrete Element (DE), the motion is integrated using rapid explicit integration schemes which require small computational time steps.

DEM calculations are very computationally-intensive, since large amounts of particles need to be considered in practical problems. On this basis, a very popular simplification for reducing the computational cost is the use of spherical particles [46] whose contact search and resolution is easier than for other geometries. This approach can be appropriate when the shape of the particles approximates spheres, or when the main focus is the macroscopic response of the material [22]. In other settings, this approach can lead to relevant inaccuracies. Several alternatives have been proposed in the literature to consider irregular DEs [23], including clusters of spheres $[44,21]$, polyhedrons $[7,14,12,29]$, spheropolyhedrons $[1,2,13,35]$ and superquadrics $[4,26,34]$, among others.

Regarding non-spherical DEs, most of the research

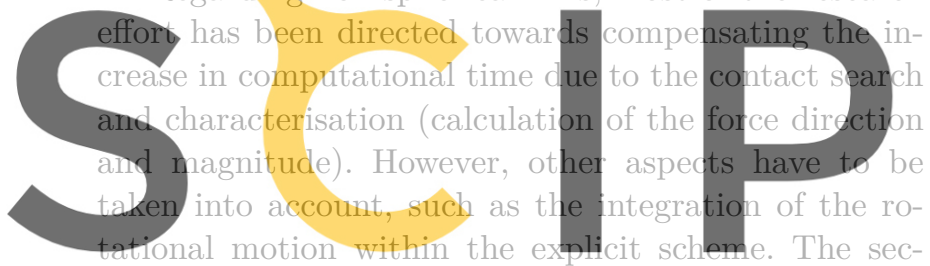

ond order inertia tensor for non-spherical particles in

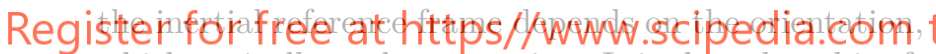

which typically evolves over time. It is clear that this affects the results, and that it depends on the integration scheme adopted. In this work, three explicit integration schemes for the rotational motion of non-spherical DEs are analysed. First, the results of their application to simple academic benchmark tests are compared. Then, two real-scale problems are considered. The approaches are evaluated in terms of their accuracy and the computational cost. In addition, the effect of the time step on both aspects is reported.

It should be noted that although we used clusters of spheres for this work (i.e. irregular particles comprised of overlapping spheres joined rigidly) [23], the results are applicable to any other irregular DE.

The rest of the article is structured as follows: first, the basis of the DEM are introduced, with more detail on the contact evaluation and the time integration. Then, a description of the integration schemes is presented, including some proposed modifications. The description of the test cases and the results obtained are provided in Section 3. Finally, overall conclusions are drawn.

\section{The Discrete Element Method}

\subsection{Contact force evaluation}

Within the DEM, the behaviour of granular materials is governed by grain-grain contact interactions [8], and the material properties are characterised through appropriate contact laws. Figure 1 shows the typical constitutive model of a contact calculated according to the DEM, where $k_{n}$ and $k_{t}$ are respectively the normal and tangential rigidities of the contact. The energy dissipation produced by the deformation at the point of contact is introduced by adding normal $\left(d_{n}\right)$ and tangential $\left(d_{t}\right)$ damping. The coefficient $\mu$ represents the friction coefficient (usually that of Coulomb).

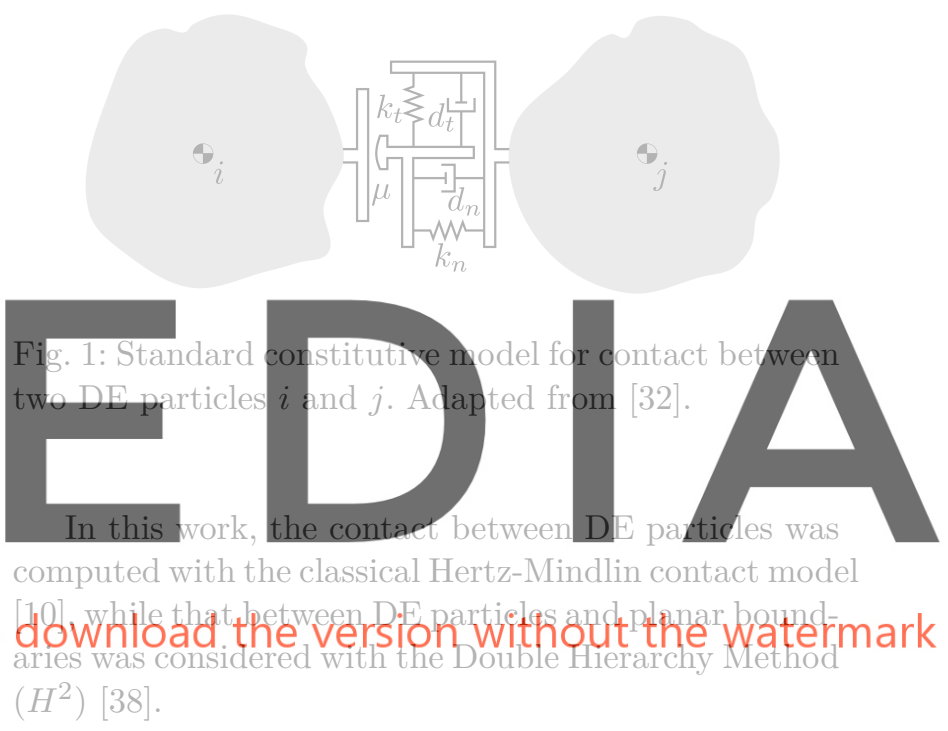

\subsection{Equations of motion}

A DE is a rigid body, e.g., the distance between two material points belonging to it remains constant over time, and its movement can be described in terms of the displacement of its centre of mass plus a rotation. In the basic DEM formulation, standard rigid body dynamic equations define the translational and rotational motion of particles. These equations can be written as:

$\mathbf{F}=m \ddot{\mathbf{u}}$

$\mathbf{T}=\mathbf{I} \dot{\omega}+\omega \times(\mathbf{I} \omega)$

where $\ddot{\mathbf{u}}$ is the particle centroid acceleration, $\boldsymbol{\omega}$ is the angular velocity, $\dot{\boldsymbol{\omega}}$ is the angular acceleration, $\mathrm{m}$ is the particle mass, $\mathbf{I}$ is the second order inertia tensor with respect to the particle centre of mass, $\mathbf{F}$ is the resultant force, and $\mathbf{T}$ is the resultant torque about the central axes. 
(F) and (T) are the outcome of summing up: (i) all forces and torques applied to the particle due to external loads, (ii) contact interaction forces and (iii) all forces and torques resulting from external damping.

The spatial description of a rigid DE can be described using two different reference frames: the inertial frame, denoted $\mathbf{X}, \mathbf{Y}$ and $\mathbf{Z}$, does not move with the DE; the element frame has its origin fixed to the centre of mass of the DE and is defined as $\mathbf{x}^{\prime}, \mathbf{y}^{\prime}$ and $\mathbf{z}^{\prime}$. Figure 2 illustrates both reference systems.

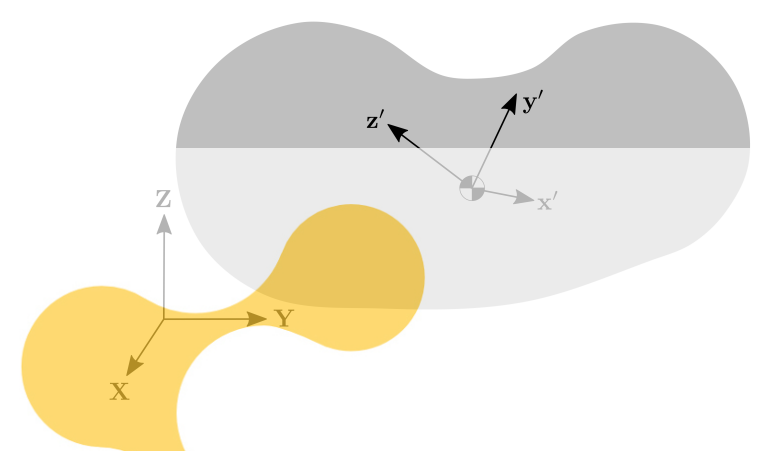

Fig. 2: Inertial XYZ and element frames of reference

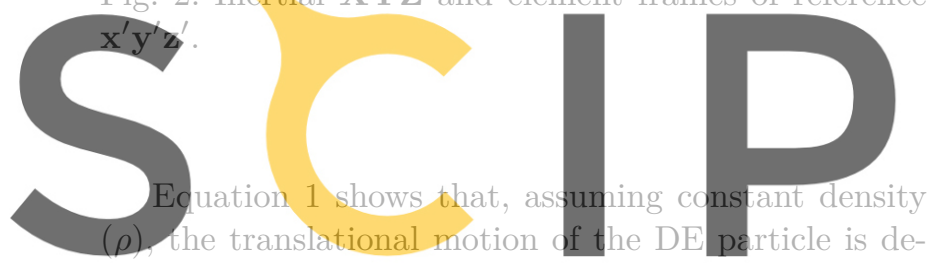

fined by its mass, which is independent of the reference

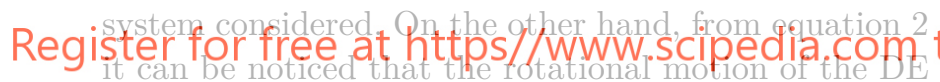

particle depends on its inertia tensor.

$\mathbf{I}=\left[\begin{array}{lll}\mathrm{I}_{x x} & \mathrm{I}_{x y} & \mathrm{I}_{x z} \\ \mathrm{I}_{y x} & \mathrm{I}_{y y} & \mathrm{I}_{y z} \\ \mathrm{I}_{z x} & \mathrm{I}_{z y} & \mathrm{I}_{z z}\end{array}\right]$

The element frame in the DEM is typically chosen to coincide with the principal axes of inertia [28], so that the products of inertia (non-diagonal components of the matrix) are zero, and the inertia tensor in the element frame is represented by a diagonal matrix:

$\mathbf{I}^{\prime}=\left[\begin{array}{ccc}\mathrm{I}_{x x}^{\prime} & 0 & 0 \\ 0 & \mathrm{I}_{y y}^{\prime} & 0 \\ 0 & 0 & \mathrm{I}_{z z}^{\prime}\end{array}\right]=\left[\begin{array}{ccc}\mathrm{I}_{x}^{\prime} & 0 & 0 \\ 0 & \mathrm{I}_{y}^{\prime} & 0 \\ 0 & 0 & \mathrm{I}_{z}^{\prime}\end{array}\right]$

This leads to the conclusion that although the expression for the translational motion (equation 1) can be efficiently resolved in the inertial reference frame, the equation defining the rotational motion (equation 2) is best expressed in the element frame (as the inertia tensor has constant components).
Equation 1 can be expressed component-wise as:

$$
\begin{aligned}
\mathrm{F}_{x} & =\mathrm{m} \ddot{\mathbf{u}}_{x} \\
\mathrm{~F}_{y} & =\ddot{m}_{y} \\
\mathrm{~F}_{z} & =\mathrm{m} \ddot{\mathbf{u}}_{z}
\end{aligned}
$$

while equation 2 component-wise in the element reference frame reads:

$$
\begin{aligned}
\mathrm{T}_{x}^{\prime} & =\mathrm{I}_{x}^{\prime} \dot{\omega}_{x}^{\prime}+\left(\mathrm{I}_{z}^{\prime}-\mathrm{I}_{y}^{\prime}\right) \omega_{z}^{\prime} \omega_{y}^{\prime} \\
\mathrm{T}_{y}^{\prime} & =\mathrm{I}_{y}^{\prime} \dot{\omega}_{y}^{\prime}+\left(\mathrm{I}_{x}^{\prime}-\mathrm{I}_{z}^{\prime}\right) \omega_{x}^{\prime} \omega_{z}^{\prime} \\
\mathrm{T}_{z}^{\prime} & =\mathrm{I}_{z}^{\prime} \dot{\omega}_{z}^{\prime}+\left(\mathrm{I}_{y}^{\prime}-\mathrm{I}_{x}^{\prime}\right) \omega_{y}^{\prime} \omega_{x}^{\prime}
\end{aligned}
$$

\subsection{Rotation operators}

An efficient procedure to transform from one reference frame to the other (and vice-versa) is essential to work with the above equations of motion. To that end, there are three alternatives (Figure 3): Euler angles, rotation matrices and quaternions [22].

The use of Euler angles leads to singularity prob-

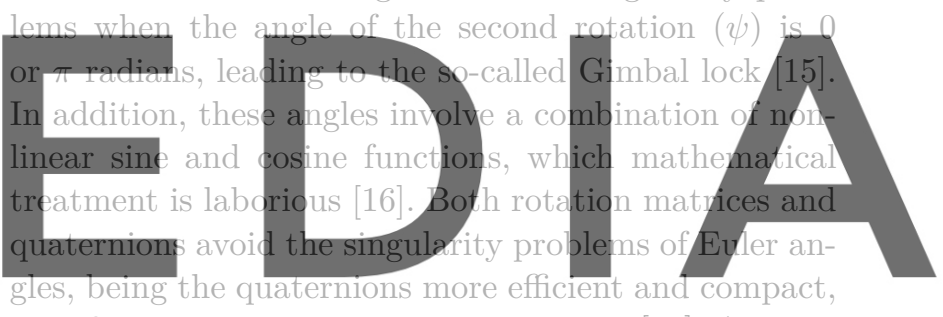

therefore with less memory requirements [24]. As a re-

o dow wal oad thae version wwithout the watermark

Quaternions can be expressed as a complex number with three imaginary components:

$q=q_{0}+q_{1} \boldsymbol{i}+q_{2} \boldsymbol{j}+q_{3} \boldsymbol{k}$

or in a compact form:

$q=\left[q_{0}, \mathbf{q}\right]$

The multiplication operation between two quaternions involves a cross product, hence it is not commutative. Quaternions $p$ and $q$ can be multiplied as:

$p q=\left[p_{0} q_{0}-\mathbf{p q}, p_{0} \mathbf{q}+q_{0} \mathbf{p}+\mathbf{p} \times \mathbf{q}\right]$

Defining the quaternion conjugate as $q^{*}=\left[q_{0},-\mathbf{q}\right]$, the norm is

$\|q\|=\sqrt{q q^{*}}$

and its inverse

$q^{-1}=\frac{q^{*}}{\|q\|^{2}}$ 


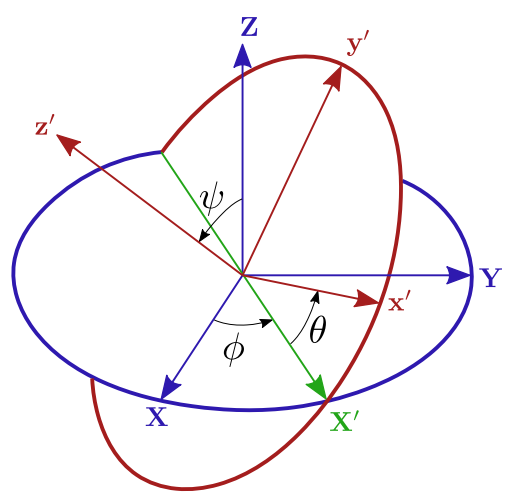

(a) Euler angles define three rotations around different axis In this case the first rotation of the coordinates system $\phi$ is around $\mathbf{Z}$, the second one $\psi$ around $\mathbf{X}^{\prime}$ and the third $\theta$ around $\mathbf{z}^{\prime}$.

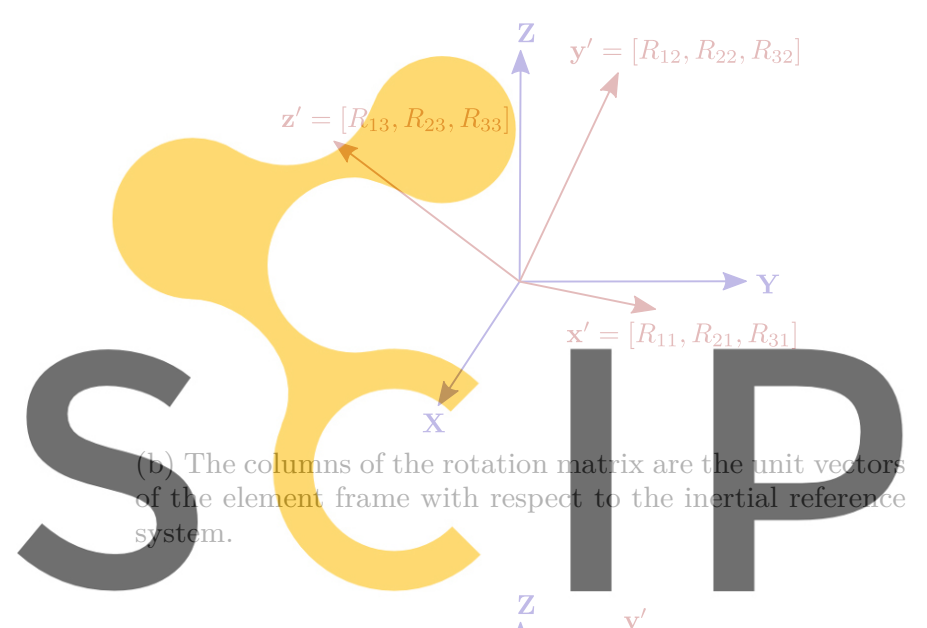

Register for free at https//wwww.scipedia.com to

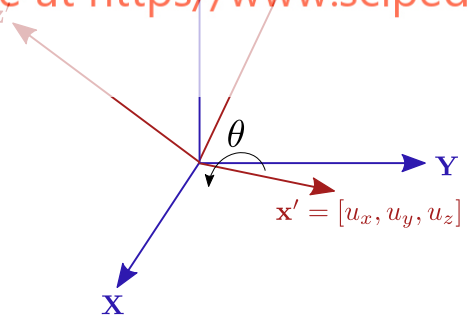

(c) Quaternions define the rotation by three components of a unit vector $u_{x}, u_{y}, u_{z}$ and a rotation $\theta$.

Fig. 3: Object orientation expressed with Euler angles, rotation matrices and quaternions. Adapted from Irazábal [22].

The transformation of vectors $\mathbf{v}$ or tensors $\mathbf{A}$ from one coordinate system to another using quaternions require them to be unitary. Otherwise, their magnitudes are not conserved after rotation. A unit quaternion is a quaternion of norm one and can be calculated dividing the original quaternion by its norm.
Considering this, the following procedure can be followed to rotate a vector $\mathbf{v}$ :

$\mathbf{v}^{\prime}=q \mathbf{v} q^{-1}$

This implies quaternion-vector multiplications which can be developed considering the vector as a quaternion with a null scalar part $\mathbf{v}=[0, \mathbf{v}]$.

The canonical way of rotating a vector $\mathbf{v}$ with a quaternion $q$ was given by equation 12 , which involves two computationally expensive quaternion multiplications. However, equation 11 can be developed in a less computationally intensive manner:

$\mathbf{v}^{\prime}=q \mathbf{v} q^{-1}=q_{0}^{2} \mathbf{v}+q_{0} \mathbf{t}+\mathbf{q} \times \mathbf{t}$

where the vector $t$ is

$\mathbf{t}=2(\mathbf{q} \times \mathbf{v})$

Regarding the rotation of second order tensors, Zhao and van Wachem [43] proposed a model for determining a tensor in the rotated framework using unit quaternions that requires that the tensor $\mathbf{A}$ is considered as three column vectors $\mathbf{A}_{1}, \mathbf{A}_{2}$ and $\mathbf{A}_{3}$ :
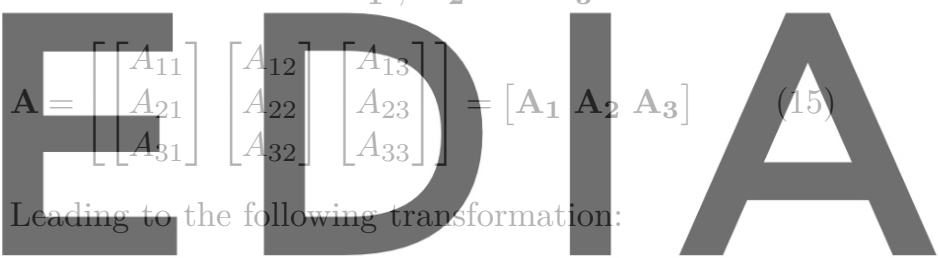

$\mathbf{A}^{\prime}=\left(q\left(q \mathbf{A} q^{-1}\right)^{T} q^{-1}\right)^{T}$

(16)

download the version without the watermark

The change of the orientation of the element frame over time can also be developed directly with quaternions by multiplying the rotational variation by the previous orientation [41]. Hereafter, the quaternions expressed in the form $q(\boldsymbol{\omega}, \Delta t)$ represent incremental rotations that are derived from the application of constant angular velocities $\boldsymbol{\omega}$ during a fraction of time $\Delta t$.

$q(\boldsymbol{\omega}, \Delta t)=\cos \left(\frac{\boldsymbol{\omega} \Delta t}{2}\right)+\sin \left(\frac{\boldsymbol{\omega} \Delta t}{2}\right) \frac{\boldsymbol{\omega}}{\|\boldsymbol{\omega}\|}$

\subsection{Time integration}

Within the DEM, equations 1 and 2 are integrated in time using an explicit scheme, where the information at the previous step is used to predict the solution at the next step. Explicit integration schemes require the time step to be below a certain limit in order to achieve computational stability. The critical time step depends on the material properties, particles geometry and constitutive model [33], though it is frequently small (between $10^{-5}-10^{-7} \mathrm{~s}$ for typical geomaterials). 
Several integration schemes of different order can be used. Low order (first and second) integration schemes are often chosen, since with such small computational time steps the use of higher order methods do not provide relevant improve in accuracy, but do imply a higher computational cost. Those methods come from the application of the Taylor series approximation of the equations that describe the problem.

\subsubsection{Translational motion}

As the aim of this work is to evaluate the effect of the rotational integration schemes, we used the Velocity Verlet [3] scheme for the translational motion. This second order predictor-corrector method provides high accuracy to compute the contact duration and the velocity and position of the particles [37] in translational motion calculations. Within the Velocity Verlet approach, the approximate velocity at instant $n+1 / 2$ is computed
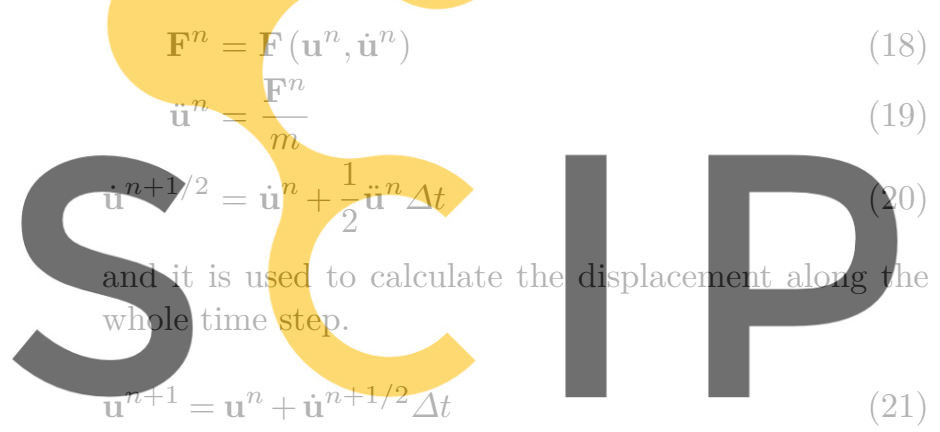

(21)
3. Quaternion integration scheme: a modification of the predictor-corrector scheme developed by Zhao and van Wachem [43], who originally proposed a direct multiplication method based on the numerical integration of unit quaternions. This method was modified to meet the corrector step (equations 22, 23 and 24) involved in the classical Velocity Verlet scheme.

These adaptations of the methods constitute an innovation of the work, making the Runge-Kutta scheme more efficient, due to the use of quaternions, and the quaternion integration scheme more accurate, adding the corrector step, as it is presented in section 3.1.2.

\section{- Direct integration scheme: the Velocity Verlet method can be extended for the rotational motion in case of spherical particles:}

$$
\begin{aligned}
& \mathrm{T}^{n}=\mathbf{T}\left(\Delta \theta^{n}, \boldsymbol{\omega}^{n}\right) \\
& \dot{\omega}^{n}=\frac{\mathbf{T}^{n}}{\mathbf{I}}
\end{aligned}
$$

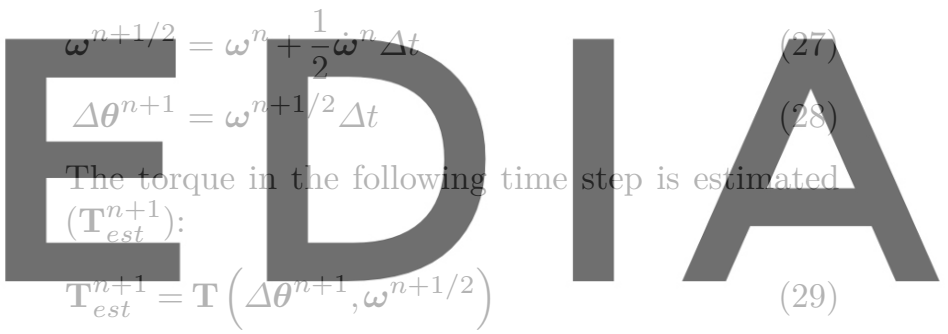

(29)

downdlodedntherversioniswithout the watermark

$\dot{\omega}^{n+1}=\frac{T_{e s t}^{n+1}}{\mathbf{I}}$

$\boldsymbol{\omega}^{n+1}=\boldsymbol{\omega}^{n+1 / 2}+\frac{1}{2} \dot{\boldsymbol{\omega}}^{n+1} \Delta t$

The rotation of spherical particles can be directly integrated using this scheme due to their symmetry, which leads to a constant second order inertia tensor in the inertial reference frame that does not depend on the orientation of the particle. Being $\mathrm{R}$ the radius of the sphere, the second order inertia tensor can be calculated as:

$\mathrm{I}=\mathrm{I}_{x}=\mathrm{I}_{y}=\mathrm{I}_{z}=\frac{2}{5} \mathrm{mR}^{2}$

By contrast, if the particles are not spherical, their second order inertia tensor in the inertial frame depends on the orientation. The angular acceleration $\dot{\omega}$ from equation 26 should by calculated in the element reference frame using the Euler equation of motion, for which the torque should be computed in the element reference frame:

$\mathbf{T}^{\prime n}=\left(q^{n}\right)^{-1} \mathbf{T}^{n} q^{n}$ 
where $q^{n}$ is the quaternion defining the transformation between the element and the inertial reference frames at time step $n$.

Now, the angular acceleration in the element reference frame reads:

$\dot{\omega}_{x}^{\prime n}=\mathrm{I}_{x}^{\prime-1}\left(\mathrm{~T}_{x}^{\prime n}-\left(\mathrm{I}_{z}^{\prime}-\mathrm{I}_{y}^{\prime}\right) \omega_{z}^{\prime n} \omega_{y}^{\prime n}\right)$

$\dot{\omega}_{y}^{\prime n}=\mathrm{I}_{y}^{\prime-1}\left(\mathrm{~T}_{y}^{\prime n}-\left(\mathrm{I}_{x}^{\prime}-\mathrm{I}_{z}^{\prime}\right) \omega_{x}^{\prime n} \omega_{z}^{\prime n}\right)$

$\dot{\omega}_{z}^{\prime n}=\mathrm{I}_{z}^{\prime-1}\left(\mathrm{~T}_{z}^{\prime n}-\left(\mathrm{I}_{y}^{\prime}-\mathrm{I}_{x}^{\prime}\right) \omega_{y}^{\prime n} \omega_{x}^{\prime n}\right)$

Knowing the angular acceleration in the element reference frame, its expression in the inertial reference frame can be directly derived from:

$\dot{\omega}^{n}=q^{n} \dot{\omega}^{\prime n}\left(q^{n}\right)^{-1}$

Then, the application of the Velocity Verlet scheme is straightforward:

$\omega^{n+1 / 2}=\omega^{n}+\frac{1}{2} \dot{\omega}^{n} \Delta t$

$\Delta \boldsymbol{\theta}^{n+1}=\omega^{n+1 / 2} \Delta t$

The estimated torque (as in equation 29) and orientation in the following time step are calculated
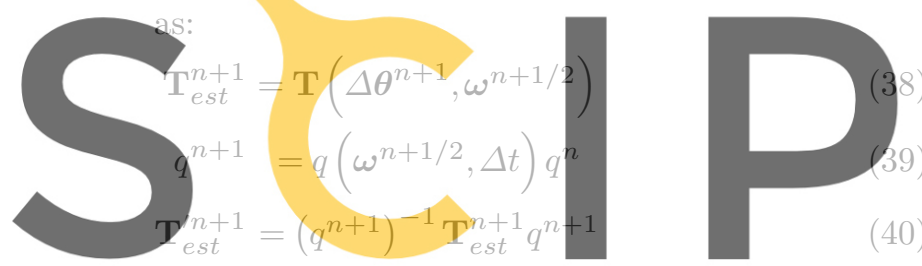

This algorithm is more accurate than the direct integration, since the angular velocities are computed through a $4^{\text {th }}$ order Runge-Kutta scheme to determine an average angular velocity $\overline{\boldsymbol{\omega}}$. To that end, four angular velocities $(\hat{\boldsymbol{\omega}})$ are estimated considering the particle with different orientations $(\hat{q})$ during the time step.

$\hat{q}_{1}=q^{n}, \hat{\boldsymbol{\omega}}_{1}=\boldsymbol{\omega}^{n}$

$\hat{q}_{2}=q\left(\hat{\boldsymbol{\omega}}_{1}, \Delta t / 2\right) q^{n}$,

$\hat{\boldsymbol{\omega}}_{2}=\left(\left(\hat{q}_{2}\left(\hat{q}_{2} \mathbf{I}^{\prime} \hat{q}_{2}^{-1}\right)^{T} \hat{q}_{2}^{-1}\right)^{T}\right)^{-1} \cdot \mathbf{H}^{n+1}$

$\hat{q}_{3}=q\left(\hat{\boldsymbol{\omega}}_{2}, \Delta t / 2\right) q^{n}$,

$\hat{\omega}_{3}=\left(\left(\hat{q}_{3}\left(\hat{q}_{3} \mathbf{I}^{\prime} \hat{q}_{3}^{-1}\right)^{T} \hat{q}_{3}^{-1}\right)^{T}\right)^{-1} \cdot \mathbb{H}^{n+1}$

$$
\begin{aligned}
& \hat{q}_{4}=q\left(\hat{\boldsymbol{\omega}}_{3}, \Delta t\right) q^{n}, \\
& \hat{\boldsymbol{\omega}}_{4}=\left(\left(\hat{q}_{4}\left(\hat{q}_{4} \mathbf{I}^{\prime} \hat{q}_{4}^{-1}\right)^{T} \hat{q}_{4}^{-1}\right)^{T}\right)^{-1} \cdot \mathbf{H}^{n+1}
\end{aligned}
$$

$$
\bar{\omega}=\frac{1}{6}\left(\hat{\omega}_{1}+2 \hat{\omega}_{2}+2 \hat{\omega}_{3}+\hat{\omega}_{4}\right)
$$

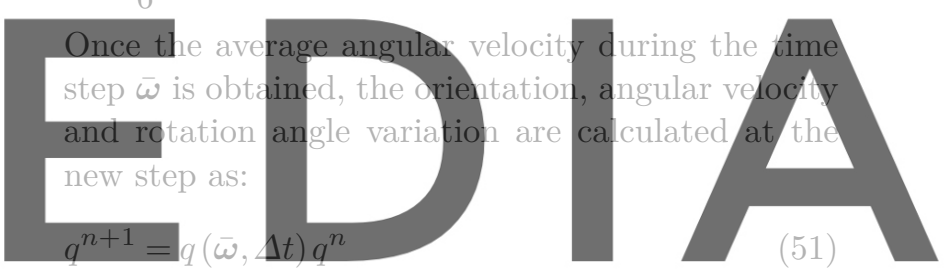

Finally, the angular velocity is updated:

$\dot{\omega}_{y}^{\prime n+1}=\mathrm{I}_{y}^{\prime-1}\left(\mathrm{~T}_{\text {est,y }}^{\prime n+1}-\left(\mathrm{I}_{x}^{\prime}-\mathrm{I}_{z}^{\prime}\right) \omega_{x}^{\prime n+1} \omega_{z}^{\prime n+1}\right)$

$\dot{\omega}_{z}^{\prime n+1}=\mathrm{I}_{z}^{\prime-1}\left(\mathrm{~T}_{e s t, z}^{\prime n+1}-\left(\mathrm{I}_{y}^{\prime}-\mathrm{I}_{x}^{\prime}\right) \omega_{y}^{\prime n+1} \omega_{x}^{\prime n+1}\right)$

$\dot{\boldsymbol{\omega}}^{n+1}=q^{n+1} \dot{\boldsymbol{\omega}}^{\prime n+1}\left(q^{n+1}\right)^{-1}$

$\boldsymbol{\omega}^{n+1}=\boldsymbol{\omega}^{n+1 / 2}+\frac{1}{2} \dot{\boldsymbol{\omega}}^{n+1} \Delta t$

- Runge-Kutta scheme: Munjiza et al. [28] considered that the changes in the angular momentum can be approximated by the increments in the external torques at every time step:

$\mathbf{H}^{n+1}=\mathbf{H}^{n}+\Delta t \mathbf{T}^{n}$

This leads to a constant angular velocity along the time step:

$\boldsymbol{\omega}^{n}=\left(\mathbf{I}^{n}\right)^{-1} \cdot \mathbf{H}^{n}=$

$\left(\left(q^{n}\left(q^{n} \mathbf{I}^{\prime n}\left(q^{n}\right)^{-1}\right)^{T}\left(q^{n}\right)^{-1}\right)^{T}\right)^{-1} \cdot \mathbf{H}^{n}$

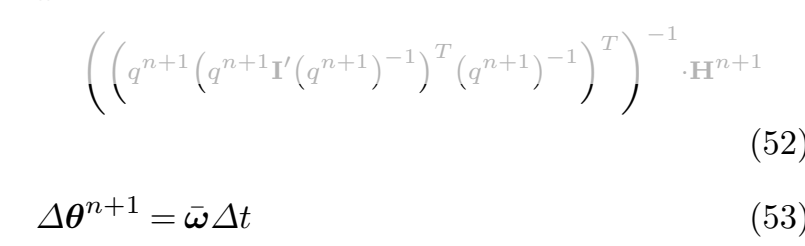

- Quaternion integration scheme: Zhao and van Wachem [43] proposed this algorithm based on directly integrating the orientations. Within this method, the variables that describe the rotational motion of the particles have to be transformed into the element frame at instant $n$ :

$\boldsymbol{\omega}^{\prime n}=\left(q^{n}\right)^{-1} \boldsymbol{\omega}^{n} q^{n}$

$\mathbf{T}^{\prime n}=\left(q^{n}\right)^{-1} \mathbf{T} q^{n}$

Now, the angular velocities at instants $n+1 / 4$ and $n+1 / 2$ are calculated as:

$\boldsymbol{\omega}^{\prime n+1 / 4}=\boldsymbol{\omega}^{\prime n}+\frac{1}{4} \dot{\omega}^{\prime n} \Delta t$

$\boldsymbol{\omega}^{\prime n+1 / 2}=\boldsymbol{\omega}^{\prime n}+\frac{1}{2} \dot{\boldsymbol{\omega}}^{\prime n} \Delta t$ 
where the angular acceleration $\dot{\boldsymbol{\omega}}^{\prime n}$ is determined using the Euler equation (equation 34). Then, the predicted angular velocity in the inertial reference frame at instant $n+1 / 4$ is approximated based on the orientation at instant $n$ :

$\boldsymbol{\omega}^{n+1 / 4}=q^{n} \boldsymbol{\omega}^{\prime n+1 / 4}\left(q^{n}\right)^{-1}$

leading to the prediction of the quaternion at instant $n+1 / 2$ :

$q^{n+1 / 2}=q\left(\boldsymbol{\omega}^{n+1 / 4}, \Delta t / 2\right) q^{n}$

which is used to calculate the angular velocity in the inertial reference frame at instant $n+1 / 2$ :

$\omega^{n+1 / 2}=q^{n+1 / 2} \omega^{\prime n+1 / 2}\left(q^{n+1 / 2}\right)^{-1}$

The variation of the rotation angle is:

$$
\Delta \theta^{n+1}=\omega^{n+1 / 2} \Delta t
$$

and the unit quaternion $q^{n+1}$ can be derived:

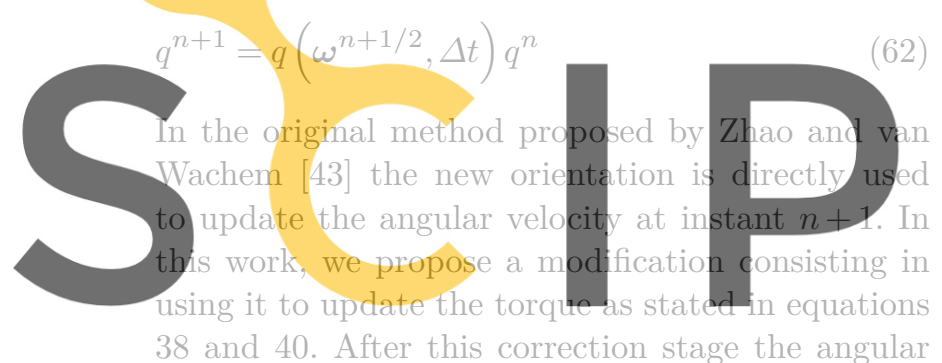

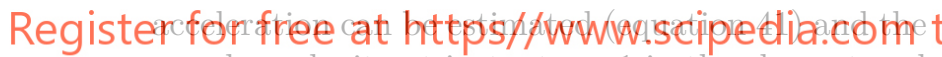

angular velocity at instant $n+1$ in the element and inertial reference frames can be updated:

$$
\begin{aligned}
\boldsymbol{\omega}^{\prime n+1} & =\boldsymbol{\omega}^{\prime n}+\dot{\boldsymbol{\omega}}^{\prime n+1} \Delta t \\
\boldsymbol{\omega}^{n+1} & =q^{n+1} \boldsymbol{\omega}^{\prime n+1}\left(q^{n+1}\right)^{-1}
\end{aligned}
$$

In the following sections, the three different methods discussed above are compared in terms of accuracy and computational time, by means of their application to different test cases.

\section{Comparison of the rotation integration schemes}

\subsection{Test cases}

This section presents two benchmark cases for which the analytical solution is known. The first was used by Munjiza et al. [28] and allows analysing the rate of convergence of each method. The second one was proposed by Zhao and van Wachem [43] to verify energy conservation.

\subsubsection{Test case 1: cylinder rotating freely}

Munjiza et al. [28] evaluated the behaviour of a cylinder freely rotating during $0.5 \mathrm{~s}$. The initial angular velocity in the inertial reference frame was set to $\omega_{0}=$ $[0.0,1.0,100.0] \mathrm{rad} / \mathrm{s}$ leading to an initial angular velocity in the element frame $\omega_{0}^{\prime}=[0.0,1.0,100.0] \mathrm{rad} / \mathrm{s}$, as shown in Figure 4.

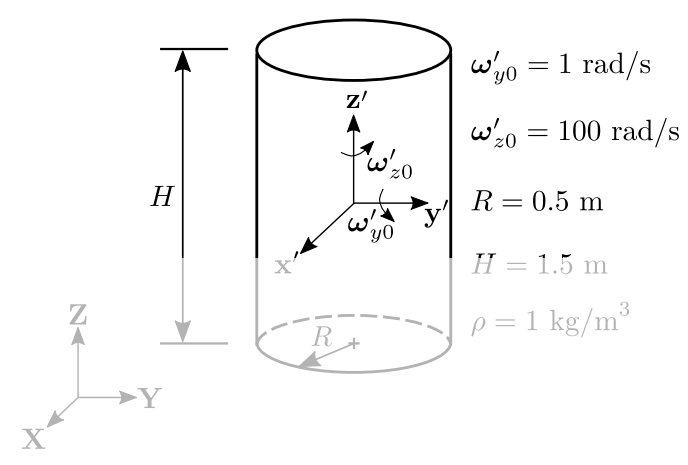

Fig. 4: Test case of a cylinder rotating freely.

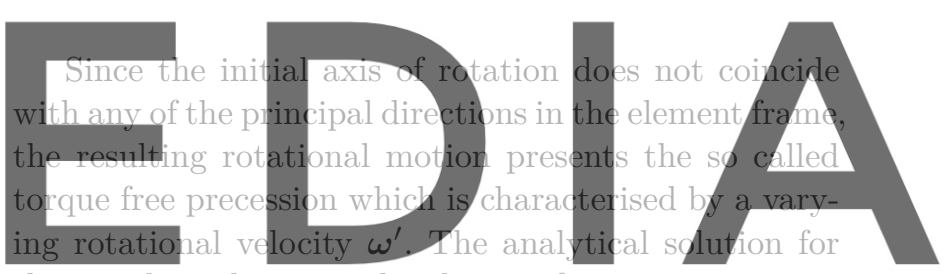

the angular velocity in the element frame is:

\section{download the version without the watermark $\omega_{y}^{\prime}=\sin (50 t) \mathrm{rad} / \mathrm{s}$ $\omega_{z}^{\prime}=100 \mathrm{rad} / \mathrm{s}$

Figures 5 and 6 show the comparison of each method with the analytical solution for the angular velocity in the axis $\mathbf{x}^{\prime}$ and $\mathbf{y}^{\prime}$ respectively. The time step of each numerical calculation is $\Delta t=10^{-3} \mathrm{~s}$ (the same used by Munjiza [28]). Results show that the direct integration scheme is highly inaccurate, with a deviation from the analytical solution that grows with time. By contrast, non-direct methods (Runge-Kutta and quaternion integration) correctly reproduce the expected outcome (numerical results overlap the analytical solution).

The same calculation was performed with different numerical time steps, to evaluate its influence in the rotational response. From the results shown in Table 1 it can be concluded that although the three schemes converge to the analytical solution with lower time steps, the rate of convergence of the direct integration scheme is much lower. Comparing the non-direct integration schemes it can be seen that the error is almost identical 

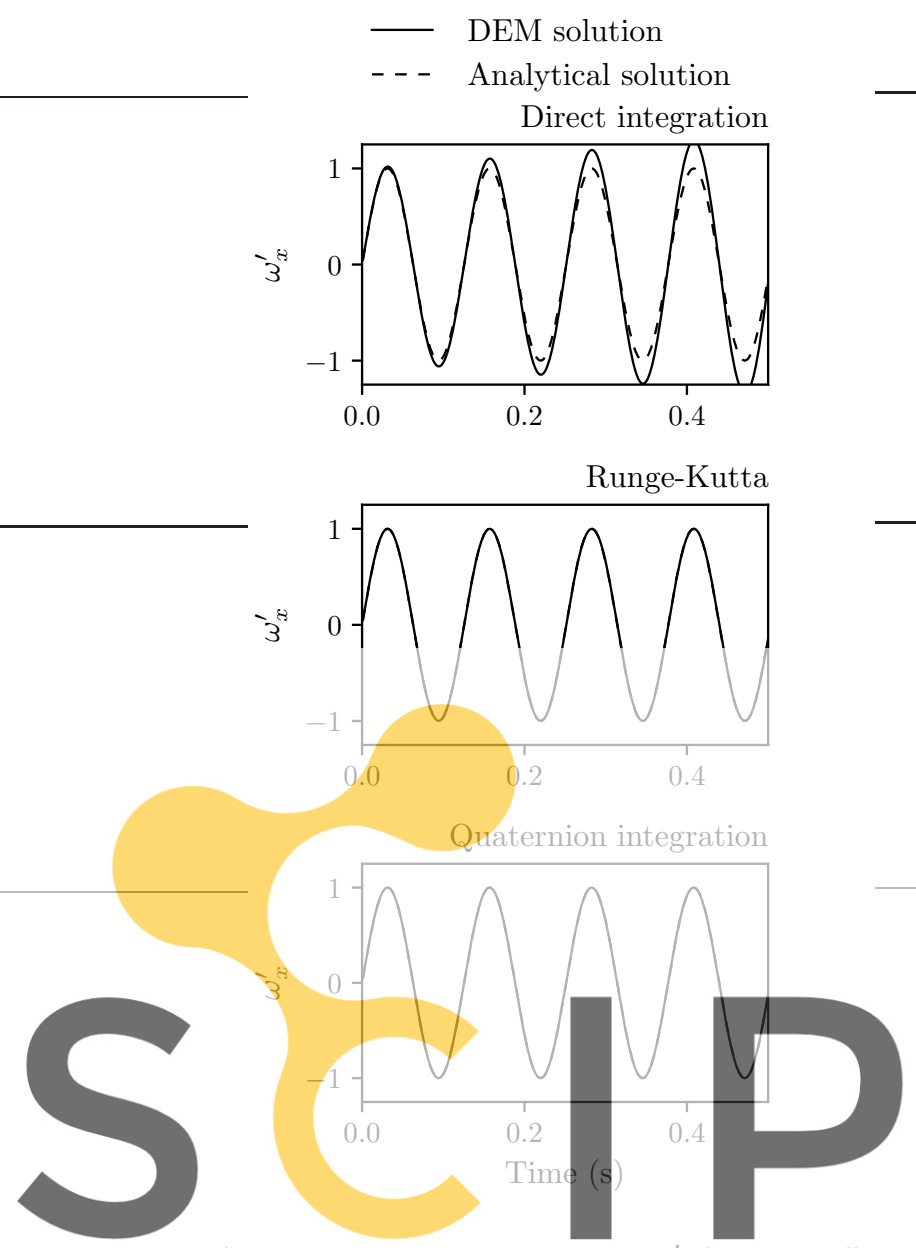

Fig. 5: Angular velocity component $\omega_{x}^{\prime}$ for the different Register for free at https//WWW.scipedia.com

till the value $\Delta t=10^{-6} \mathrm{~s}$ for which the quaternion integration scheme behaves slightly better. However, for such small errors the differences may be caused by numerical issues.

These results may seem contrary to the theoretical framework indicating that the error decays faster for higher order schemes, but it should be considered that the quaternion integration scheme $\left(2^{\text {nd }}\right.$ order) includes two additional predictor-corrector steps for the angular velocity (equations 60 and 64) in contrast to the RungeKutta scheme ( $4^{\text {th }}$ order). Comparing the rate of convergence of the quaternion and the direct integration schemes, both of $2^{\text {nd }}$ order, the rate of convergence of the first one is higher because, on top of that, it carries out all additions and subtractions concerning angular velocities in the elemental reference frame, not in the absolute one that may lead to errors.

In this section, the new quaternion integration scheme was not compared with the classical one as they would produce identical results: the improvement proposed is
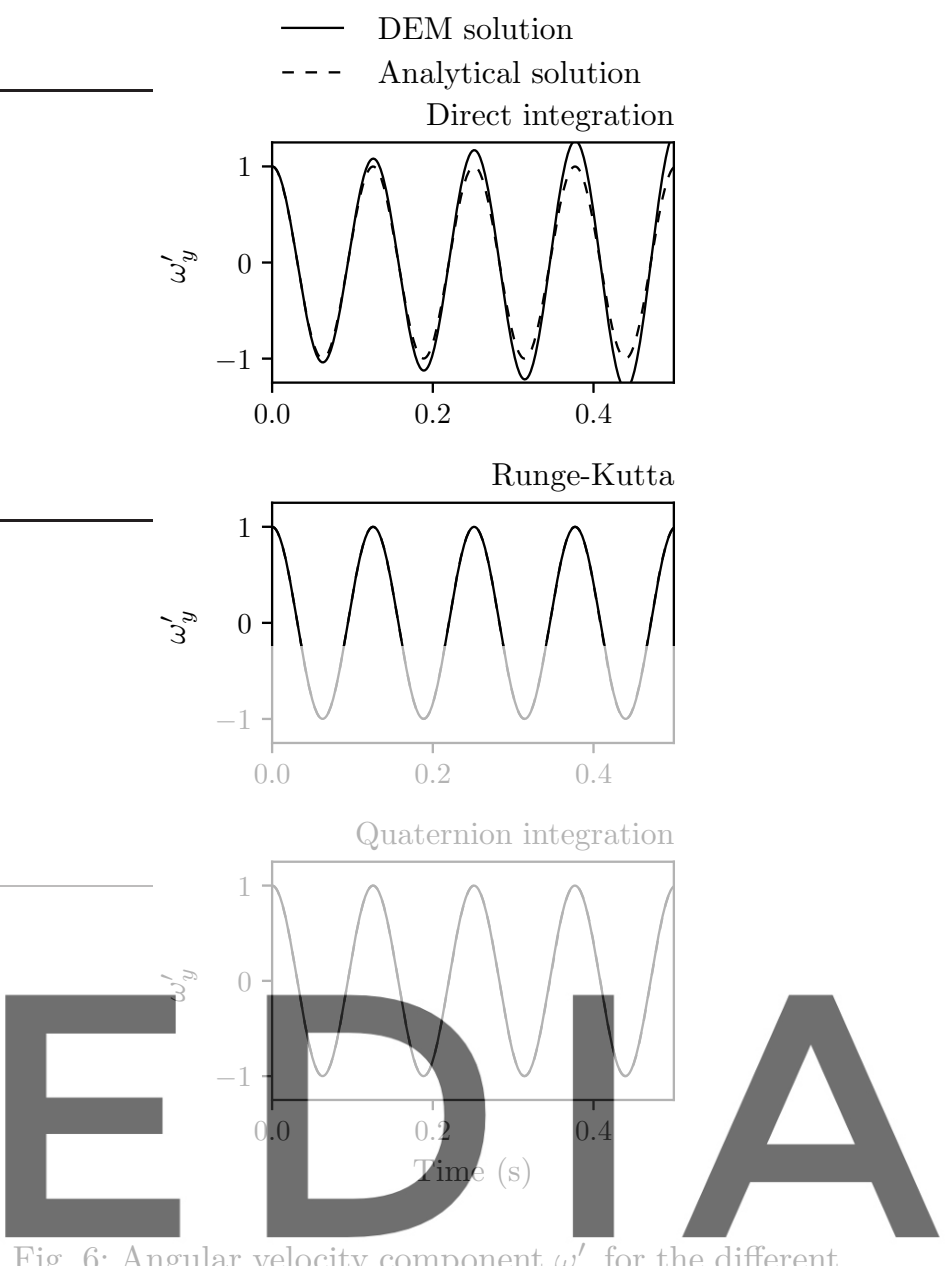

Fig. 6: Angular velocity component $\omega_{y}^{\prime}$ for the different rotational inteoration schemes and $\Delta t=10^{-3} \mathrm{~s}$ download the version without the watermark

Table 1: Mean squared error of each scheme for different time steps.

\begin{tabular}{c|c|c|c}
\multicolumn{2}{l|}{} & Error $\omega_{x}^{\prime}$ & Error $\omega_{y}^{\prime}$ \\
\hline \multirow{3}{*}{$\Delta t=10^{-2}$} & Direct int. & $6.64 \cdot 10^{-1}$ & $6.68 \cdot 10^{-1}$ \\
& Runge-Kutta & $3.90 \cdot 10^{-1}$ & $4.09 \cdot 10^{-1}$ \\
& Quaternion int. & $4.94 \cdot 10^{-1}$ & $4.78 \cdot 10^{-1}$ \\
\hline \multirow{3}{*}{$\Delta t=10^{-3}$} & Direct int. & $2.79 \cdot 10^{-1}$ & $2.77 \cdot 10^{-1}$ \\
& Runge-Kutta & $4.23 \cdot 10^{-3}$ & $4.29 \cdot 10^{-3}$ \\
& Quaternion int. & $4.23 \cdot 10^{-3}$ & $4.29 \cdot 10^{-3}$ \\
\hline \multirow{3}{*}{$\Delta t=10^{-4}$} & Direct int. & $3.72 \cdot 10^{-2}$ & $3.68 \cdot 10^{-2}$ \\
& Runge-Kutta & $4.23 \cdot 10^{-5}$ & $4.28 \cdot 10^{-5}$ \\
& Quaternion int. & $4.23 \cdot 10^{-5}$ & $4.28 \cdot 10^{-5}$ \\
\hline \multirow{3}{*}{$\Delta t=10^{-5}$} & Direct int. & $3.84 \cdot 10^{-3}$ & $3.79 \cdot 10^{-3}$ \\
& Runge-Kutta & $4.22 \cdot 10^{-7}$ & $4.28 \cdot 10^{-7}$ \\
& Quaternion int. & $4.22 \cdot 10^{-7}$ & $4.28 \cdot 10^{-7}$ \\
\hline \multirow{3}{*}{$\Delta t=10^{-6}$} & Direct int. & $3.85 \cdot 10^{-4}$ & $3.80 \cdot 10^{-4}$ \\
& Runge-Kutta & $2.95 \cdot 10^{-8}$ & $2.99 \cdot 10^{-8}$ \\
& Quaternion int. & $4.30 \cdot 10^{-9}$ & $4.36 \cdot 10^{-9}$
\end{tabular}


based on calculating an estimated torque at the end of each time step, and no torque is applied in this test.

So now comes the question, considering the small time steps used in the classical DEM calculations, is it worth using the non-direct integration schemes? This is analysed in the following tests.

\subsubsection{Test case 2: single fibre bouncing}

In this test, a single fibre falls down under the effect of gravity. The particle used is a cluster comprised by five spheres representing the fibre shown in Figure 7. The aim is to analyse energy conservation for the different integration schemes.

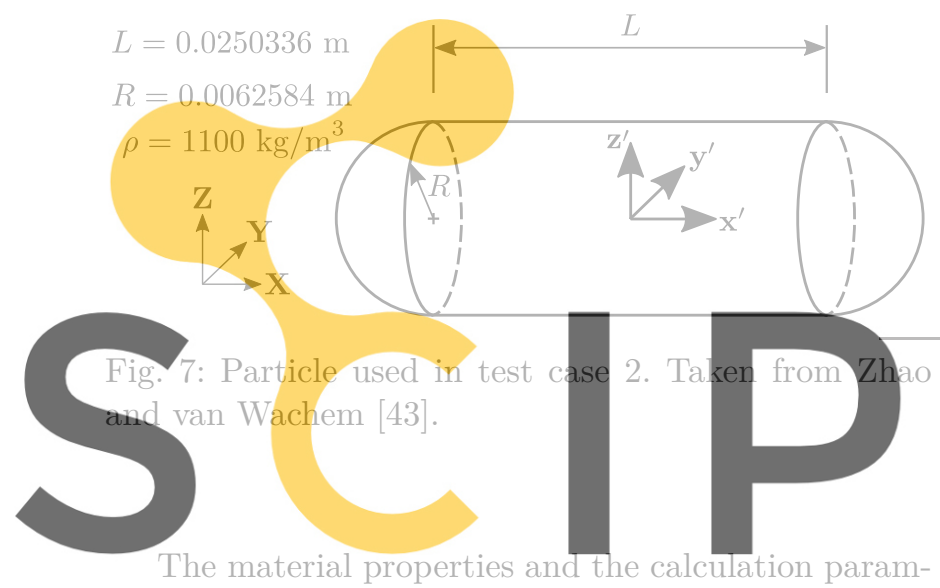
eters of this test are summarised in Table 2. The friction

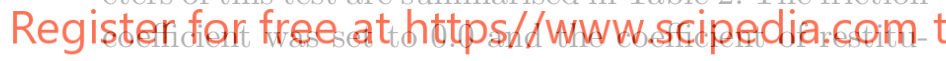
tion to 1.0, in order to avoid energy losses due to friction and damping

Table 2: Material properties and calculation parameters used in test case 2 .

\begin{tabular}{lc}
\hline Young modulus of the fibre $(\mathrm{Pa})$ & $5 \cdot 10^{7}$ \\
Poisson ratio of the fibre & 0.35 \\
Young modulus of the walls $(\mathrm{Pa})$ & $5 \cdot 10^{7}$ \\
Poisson ratio of the walls & 0.23 \\
Friction coefficient (all contacts) & 0.0 \\
Restitution coefficient (all contacts) & 1.0 \\
Time step (s) & $10^{-7}$ \\
\hline
\end{tabular}

Initially, the axis $\mathbf{x}^{\prime}$ of the fibre is oriented in the direction $[0.86603,-0.5,0.0]$ of the inertial frame and its centre of gravity is located $0.5 \mathrm{~m}$ over the horizontal floor. As a result, its collision with the floor induces a torque to the fibre, which in turn generates a rotation.
This allows the evaluation of the rotational kinetic energy, which can be used for assessing the correctness of each rotational integration scheme. This test involves contact with a boundary, therefore represents better practical problems.

The energy of the fibre along the calculation is presented in Figure 8 for the three integration schemes. As neither friction nor damping is applied, the total energy is expected to be conserved. The kinematic, gravitational and elastic energy are also separately plotted. It can be seen that the elastic energy only appears when the fibre is in contact with the floor. Although the total energy of the fibre is correctly conserved along the whole calculation, relevant differences can be seen regarding the kinetic and gravitational energy between the direct integration scheme and the two non-direct ones. Also, while the particle bounces three times with the former method, four contacts are recorded for the latter approaches during the calculation.
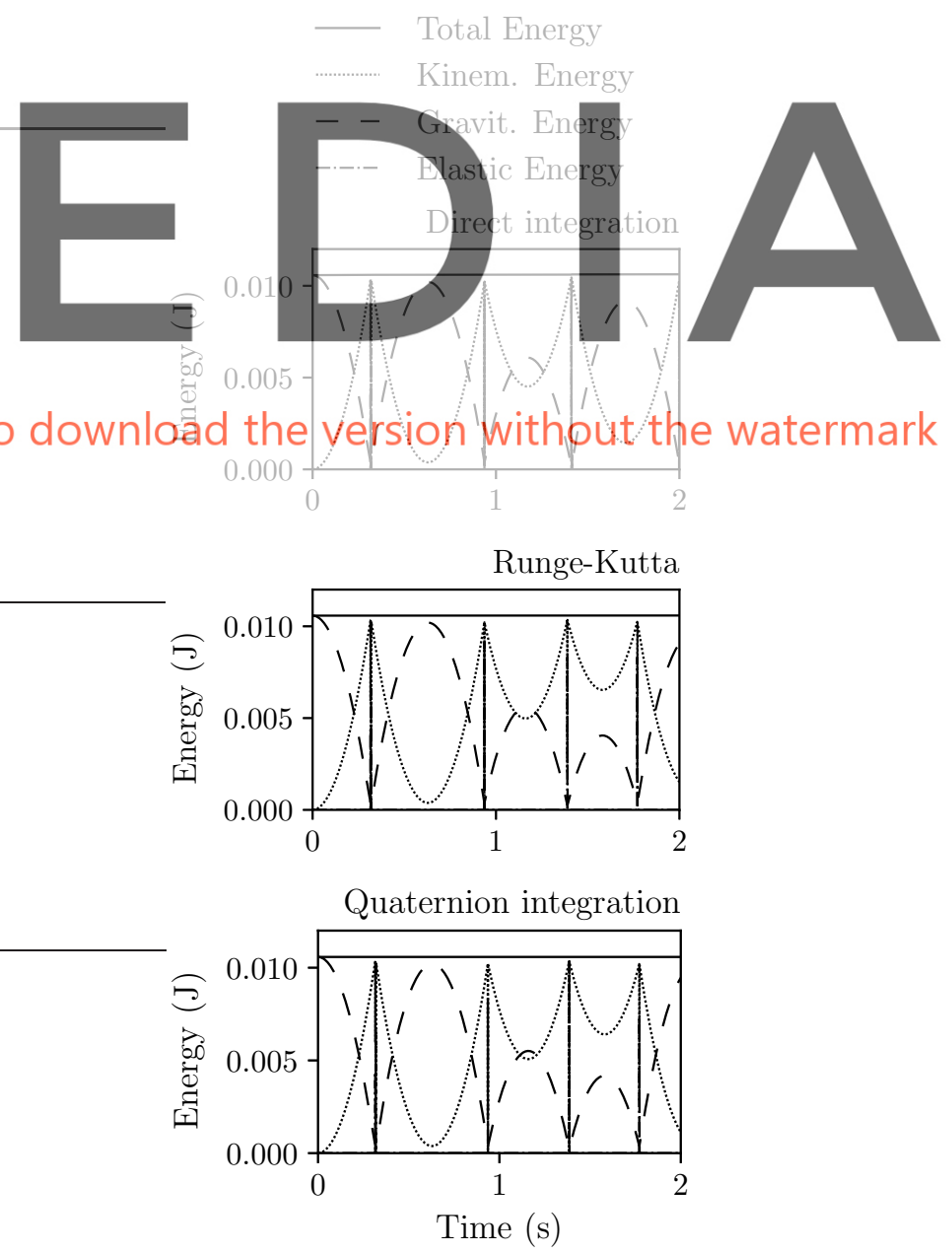

Fig. 8: Energy vs. time for the different rotation integration schemes $\left(\Delta t=10^{-7} \mathrm{~s}\right)$. 
Figure 9 shows the error in the total energy as a function of time for the different integration schemes. The error using the direct method is almost $0.4 \%$ at the end of the calculation, while it remains below $0.1 \%$ for Runge-Kutta and even lower for the Zhao and van Wachem scheme. It is interesting to note that the change in total energy occurs at bounces, when torque is applied. This is in accordance with the results of the first test case: when torque is not relevant, all three methods converge to the analytical solution with small time step.
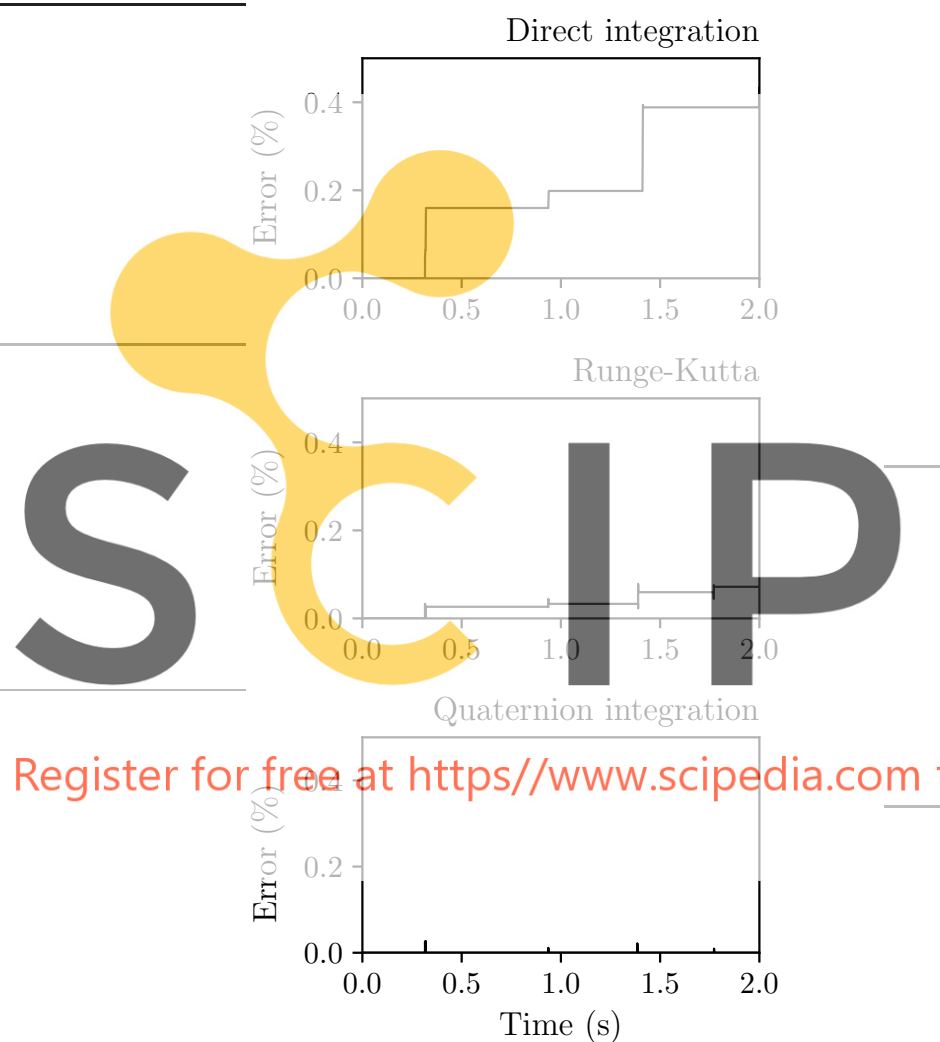

Fig. 9: Error in the energy conservation between the different rotation integration schemes $\left(\Delta t=10^{-7} \mathrm{~s}\right)$.

These results show that, when contact evaluation is relevant, time integration schemes can be inaccurate even for small time steps. In this case, the quaternion integration scheme is better as the corrector step increases the accuracy. Moreover, the quaternion integration scheme presented in this work has been compared with the classical Zhao and van Wachem scheme. The results of the error in the energy evaluation are presented in Figure 10. It can be clearly seen that the improvements introduced in this work increases the accuracy.

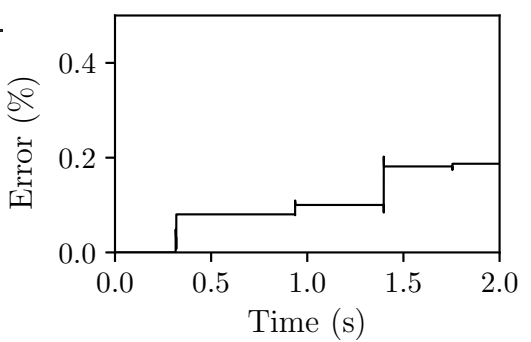

Fig. 10: Error in the energy conservation of the classical quaternion integration scheme developed by Zhao and van Wachem $\left(\Delta t=10^{-7} \mathrm{~s}\right)$.

\section{Finally, the test was performed with a higher time} step $\Delta t=10^{-5} \mathrm{~s}$, trying to evaluate the robustness of each scheme. The error in the energy evaluation can be seen in Figure 11 where the superiority of the quaternion integration scheme can be clearly appreciated.
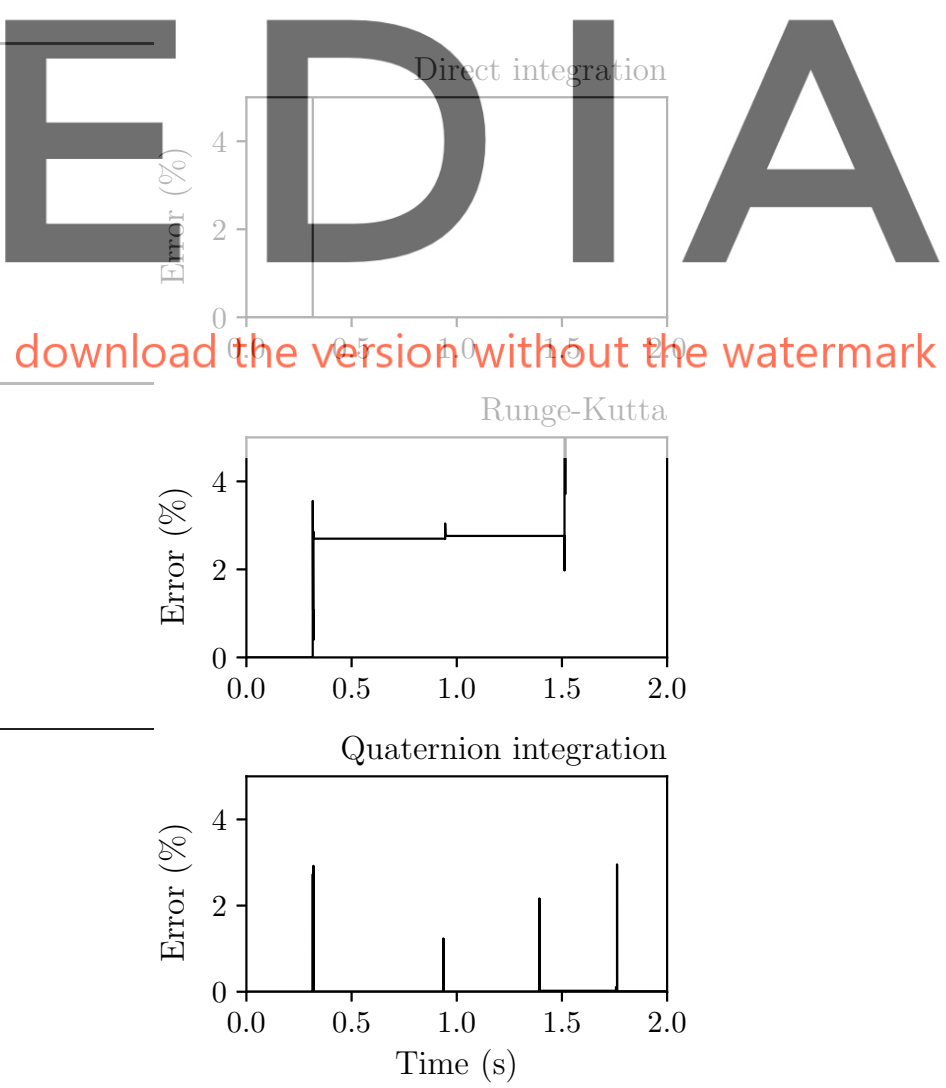

Fig. 11: Error in the energy conservation between the different rotation integration schemes $\left(\Delta t=10^{-5} \mathrm{~s}\right)$. 


\subsection{Real tests}

The accuracy of each of the rotational integration schemes have already been shown for individual particles. However, real-case problems typically involve high amount of DEs. Therefore, small differences in benchmark cases for single particles can result in high deviations from the expected behaviour in practical problems. This is why two calculations representing classical real-scale experimental tests for granular materials are presented in this section. The first one is the discharge of a silo full of irregular particles. This test is highly dynamic, so it allows to check if the differences between integration schemes affect the response of the particles when their relative velocity is high. The second test represents a direct shear test where the particles movement is not so important.

In both calculations, the particles used are those shown in Figure 12: 6 irregular clusters comprising between 29 and 45 spheres. Those particles are randomly displayed all over the computational domain and their size is between 30 and $65 \mathrm{~mm}$. These geometries represent railway ballast stones, a granular material whose calculation with the DEM is very extended. The material properties and calculation parameters for both calculations (Table 3 ) were also chosen to emulate railway ballast [22].
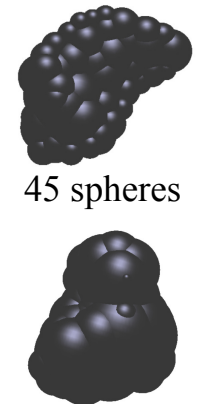

33 spheres

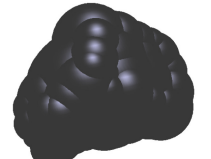

44 spheres

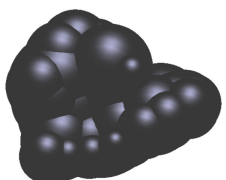

43 spheres

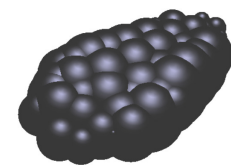

45 spheres

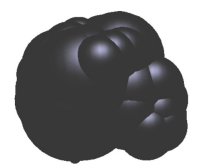

29 spheres
Fig. 12: Geometry of the particles used for the calculations.

\subsubsection{Test case 3: silo discharge}

The discharge of silos is a difficult engineering problem typically modelled with the DEM [40, 42]. The problem definition is based on that proposed by Chen et al. [5], however, in this case the amount of material is higher. The aim of the original test was to measure the angle of repose [39] of railway ballast. The geometry of the calculation is presented in Figure 13.
Table 3: Material properties and calculation parameters used in test cases 3 and 4 .

\begin{tabular}{lc}
\hline Density of the DE particles $\left(\mathrm{kg} / \mathrm{m}^{3}\right)$ & 2700 \\
Young modulus (all contacts) $(\mathrm{Pa})$ & $2.4 \cdot 10^{8}$ \\
Poisson ratio (all contacts) & 0.2 \\
Friction coefficient (between particles) & 0.6 \\
Friction coefficient (particle-silo) & 0.0 \\
Friction coefficient (particle-floor) & 0.6 \\
Restitution coefficient (all contacts) & 0.0 \\
Time step (s) & $10^{-6}$ \\
\hline
\end{tabular}

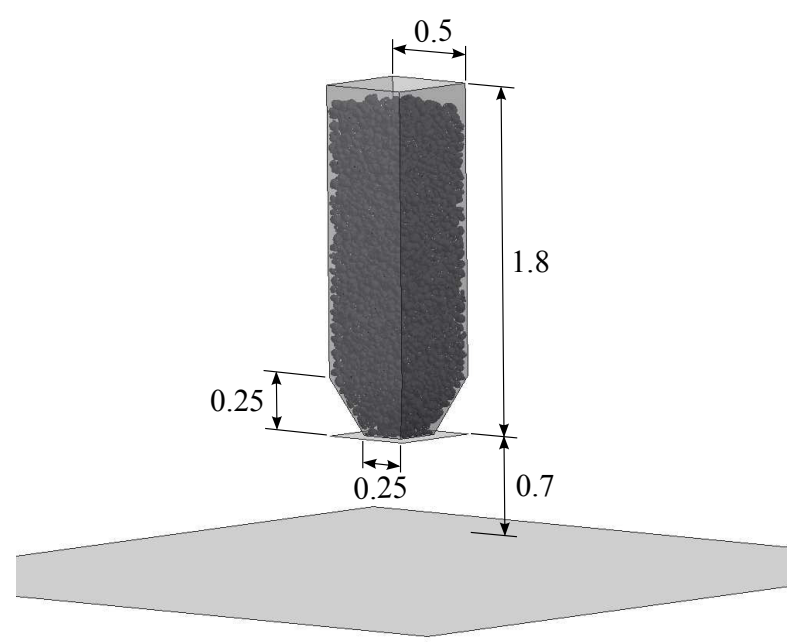

Fig. 13: Calculation layout (dimensions in $\mathrm{mm}$ ). Adapted from Chen et al. [5].

Figure 14 shows the evolution of the material flowing for the three schemes. When the calculation time is $6.0 \mathrm{~s}$ it can be seen that the discharge is slower with the direct integration scheme (the amount of material remaining in the silo is greater) than for the non-direct schemes. Also, small differences were obtained in the final shape of the pile.

As this is the test involving the largest amount of particles in this work (179978 spheres in 4497 clusters), it was used to asses the computational time of the three integration schemes. Table 4 summarises the discharge time, angle of repose and computational time per second of calculation for each case. Since the quaternion integration scheme showed to be more accurate in test case 2 (section 3.1.2), it was taken as the reference for comparison with the direct integration and RungeKutta.

The difference in the discharge time and angle of repose is over the $2 \%$ between the direct and quaternion integration schemes, while between the Runge-Kutta 


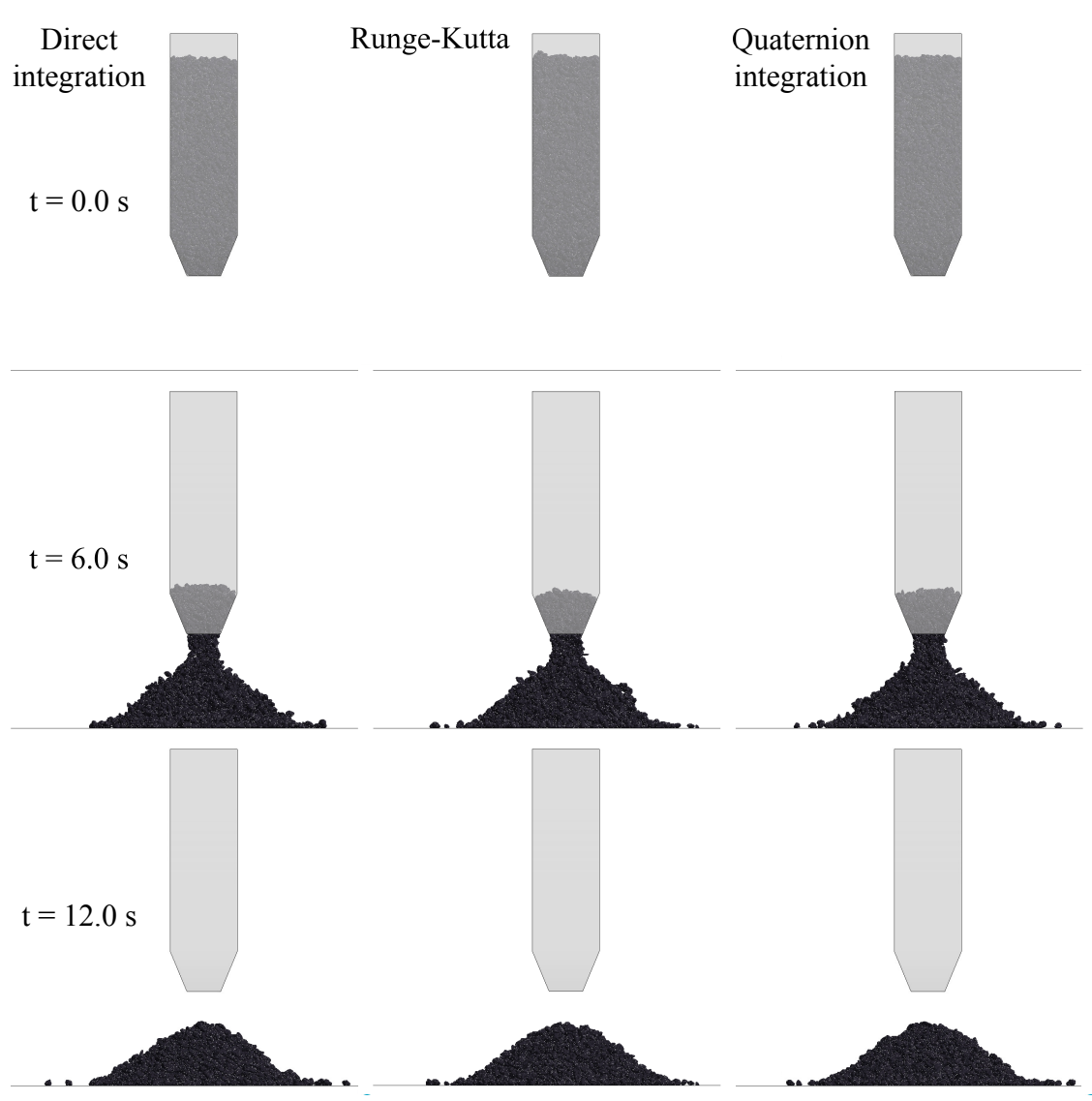

Fig. 14: Evolution of the calculation for the three schemes.

Table 4: Discharge time, angle of repose and computational time per second of calculation for each scheme. To calculate the difference between the schemes the quaternion integration scheme was chosen as the reference.

\begin{tabular}{c|cc|cc|cc|} 
& \multicolumn{2}{|c|}{ Discharge time } & \multicolumn{2}{c|}{ Angle of repose } & \multicolumn{2}{c|}{ Computational time } \\
& Time (s) & Difference (\%) & Angle (degrees) & Difference (\%) & Time (h) & Difference (\%) \\
\hline Direct Integration & 6.90 & 2.22 & 35.8 & 4.78 & 17.77 & 0.23 \\
Runge-Kutta & 6.70 & 0.74 & 37.3 & 0.80 & 17.76 & 0.19 \\
Quaternion Integration & 6.75 & - & 37.6 & - & 17.73 & -
\end{tabular}

and quaternion integration schemes is lower than $1 \%$ for both parameters. The angle of repose was the average of four measurements at different locations in the granular cone [25]. Regarding the computational time, the difference between the quickest (quaternion integration) and the slowest (direct integration) calculation was about $0.2 \%$, which can be considered as negligible. The calculations were developed in an Intel Xeon E52670 with 16 processors.

From these results it can be concluded that for highly dynamic cases involving a large amount of irregular particles the use of non-direct rotational integration schemes is advisable, as they provide more accurate results without increasing the computational time. More- over, although the differences between the Runge-Kutta and quaternion integration schemes is low, we recommend the use of the quaternion integration scheme which has been found to be more accurate and robust in test case 2 .

\subsubsection{Test case 4: Direct shear test}

In the direct shear test, a sample of granular material is introduced in a box divided in two halves. Then the lower one is pulled and the stress-strain curve is recorded. Among other authors, this test was documented by Indraratna et al. [20] and Huang and Tutumluer [17]. The geometry of the box used in the nu- 
merical model (Figure 15) is based in the laboratory tests carried out at the University of Wollongong in $2011[20]$.

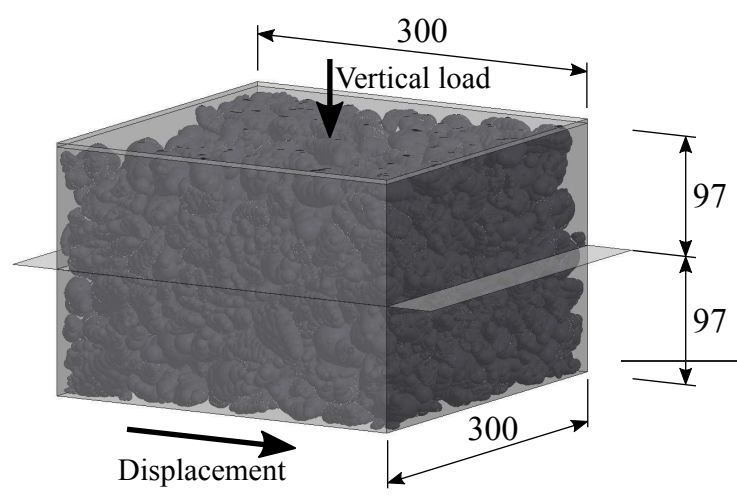

Fig. 15: Numerical model of the device used in the largescale box shear tests (dimensions in $\mathrm{mm}$ ).

The direct shear test is useful for evaluating the behaviour of granular materials under various confinement pressures. In this work, it is used to analyse the performance of the three rotational integration schemes in a quasi-static situation, when the material is subjected to three different confinement pressures: 27, 51 and $75 \mathrm{kPa}$. The lower box moves with a velocity equal to $0.0005 \mathrm{~m} / \mathrm{s}$.

The material properties and calculation parameters used in these calculations are those presented in Table 3 , as in the previous case. Regarding the contact constitutive law, former works, such as Irazabal [22] and Hohner et al. [18], described the problem of representing confined granular material samples with clusters. The overlapping spheres introduce a geometric friction and imply discontinuity of contacts; complex contact constitutive laws, difficult to calibrate, should be applied [22] to minimise these effects. As this work focuses on the integration schemes, the classical Hertz contact law has been used.

The results shown in Figure 16 present the shear stress as a function of the displacement of the lower box for the three confinement pressures. As this test is almost quasi-static, it may be assumed that the differences between the three methods would be small. However, results show a high deviation between the direct and non-direct integration methods even in this case. On the other hand, the deviation between the RungeKutta and the quaternion integration schemes is low. Table 5 shows the relative difference at the end of the calculation between the results obtained with the direct integration and Runge-Kutta schemes compared to the quaternion integration scheme.
- Direct Integration

........ Runge-Kutta

-.-. Quaternion Integration

$27 \mathrm{kPa}$
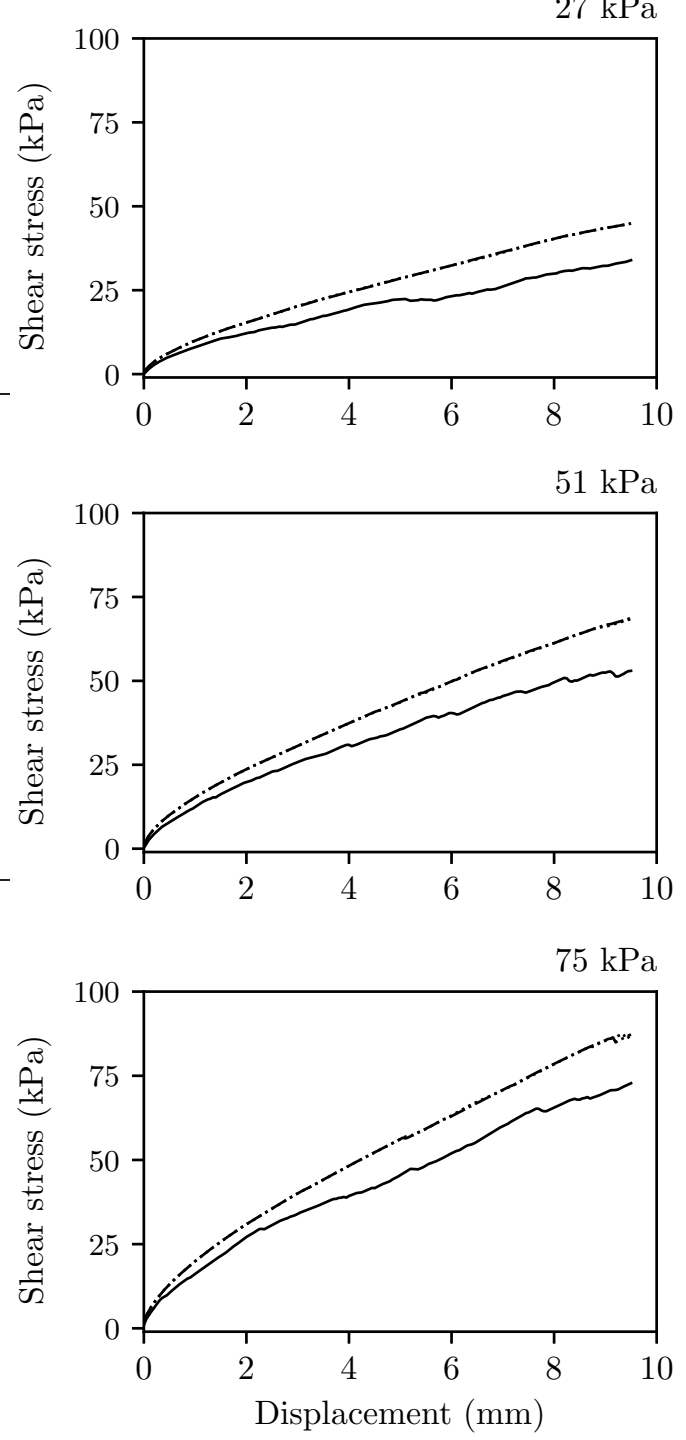

Fig. 16: Results of the direct shear tests obtained with each of the integration schemes. Runge-Kutta and quaternion integration results are overlapped.

Table 5: Relative difference in shear stress compared to the quaternion integration scheme at the end of the calculation.

\begin{tabular}{c|c|c} 
& Direct integration & Runge-Kutta \\
\hline $27 \mathrm{kPa}$ & $23.37 \%$ & $0.59 \%$ \\
$51 \mathrm{kPa}$ & $22.94 \%$ & $0.47 \%$ \\
$75 \mathrm{kPa}$ & $15.94 \%$ & $0.26 \%$
\end{tabular}


Results show that non-direct integration schemes should also be applied even in quasi-static situations, when non-spherical particles are considered.

\section{Summary and conclusions}

In this work, the performance of three different schemes for integrating the rotational motion of irregular particles within the DEM are evaluated. Although the text is focused in the application of the algorithms to the DEM, these methods can be adapted for any computational method which requires the explicit integration of the rotational motion.

It is well known that the integration of the rotation with the first and second order schemes based on the Taylor series approximation leads to inaccuracies when working with non-spherical particles. This is due to the influence of the orientation of the particles in the second order inertia tensor in the inertial reference frame.

We considered three integration approaches: a direct explicit integration based on the Velocity Verlet scheme, and two non-direct schemes: a $4^{\text {th }}$ order RungeKutta firstly developed by Munjiza et al. [28], and a method based on the numerical integration of unit quaternions presented by Zhao and van Wachem [43]. We proposed an improved version of the latter, where a Velocity Verlet scheme is used for translation.

In the first analytical benchmark, which involves extremely fast rotation, the direct explicit integration approach provided results with error increasing with time with respect to the analytical solution. Both non-direct schemes showed similar performance in terms of accuracy of calculation of the angular velocity, as well as of computational time. The same results were obtained for different time steps.

In the second benchmark, results were evaluated in terms of the energy conservation, since contact plays a key role. Results were similar, with a slightly better performance of the quaternion integration (lower than $0.1 \%$ ). Here, the inaccuracy of the direct integration was clearly observed, since the particle bounced three times in the time considered, whereas 4 bounces were recorded for the non-direct schemes. Interestingly, the improvement proposed with respect to the original version by Zhao and van Wachem resulted in a better energy conservation $(0.2 \%$ in the period of analysis, though increasing with time).

The small differences observed between the nondirect schemes in the benchmark cases, where a single particle was considered, could in principle become very relevant in real cases, in which high amount of particles are typically involved. To verify this aspect, the same comparison was performed in models representing real laboratory tests, with thousands of particles. Again, non-direct schemes provided similar results, and clearly outperformed the direct integration. This behaviour was observed even in the direct shear test, where rotation is less relevant because of its quasistatic nature.

However, the results from the non-direct schemes were almost identical with regard to the macroscopic variables considered: angle of repose and discharge time for the silo test, and shear stress in the box test. This means that other differences between the benchmark cases and the real ones compensate for the effect of the number of elements. Indeed, some aspects of the real cases imply that the rotation integration is less important: a) the coefficient of restitution is much lower in the real case; b) the clusters of spheres that represent the ballast stones, though irregular, resemble more of spheres than the particles of the benchmarks, and c) the rotation velocity is much lower in real cases.

These results suggest that the 2 non-direct methods can be used indistinctly in real cases. Nonetheless, we recommend using the Zhao and van Wachem method with the modification proposed in this work, since it provides better energy conservation with equal computational cost. This difference could be relevant in industrial or engineering processes in which the integration of rotation is more relevant, because of the shape of the particles, of their velocity of rotation, or some other aspect.

On behalf of all authors, the corresponding author states that there is no conflict of interest.

Acknowledgements This work was carried out with the financial support Spanish MINECO within the MONICAB (BIA201567263-R) project.

\section{References}

1. Alonso-Marroquín F (2008) Spheropolygons: A new method to simulate conservative and dissipative interactions between $2 \mathrm{~d}$ complex-shaped rigid bodies. EPL 83(1):14001

2. Alonso-Marroquín F, Wang Y (2009) An efficient algorithm for granular dynamics simulations with complex-shaped objects. Granular Matter 11(5):317-329

3. Belytschko T, Liu WK, Moran B, Elkhodary K (2013) Nonlinear finite elements for continua and structures. John wiley \& sons

4. Chakraborty N, Peng J, Akella S, Mitchell JE (2008) Proximity Queries Between Convex Objects: 
An Interior Point Approach for Implicit Surfaces. IEEE T Robot 24(1):211-220

5. Chen C, McDowell GR, Thom NH (2014) Investigating geogrid-reinforced ballast: Experimental pull-out tests and discrete element modelling. Soils and Foundations 54(1):1-11

6. Chen W, Qiu T (2012) Numerical Simulations for Large Deformation of Granular Materials Using Smoothed Particle Hydrodynamics Method. International Journal of Geomechanics 12(2):127-135

7. Cundall PA (1988) Formulation of a threedimensional distinct element model-Part I. A scheme to detect and represent contacts in a system composed of many polyhedral blocks. International Journal of Rock Mechanics and Mining Sciences 25(3):107-116

8. Cundall PA, Strack ODL (1979) A Discrete Numerical Model for Granular Assemblies. Géotechnique 29(1):47-65

9. Dafalias Y, Manzari M (1997) A Critical State Twosurface Plasticity Model for Sands. Géotechnique 47(2):255-272

10. Deresiewicz H, Mindlin RD (1952) Elastic spheres in contact under varying oblique forces. Columbia University. Department of Civil Engineering.

11. Duncan J (1996) State of the Art: Limit Equilibrium and Finite-Element Analysis of Slopes. J Geotech Eng-ASCE 122(7):577-596

12. Eliáš J (2014) Simulation of railway ballast using crushable polyhedral particles. Powder Technol 264:458-465

13. Galindo-Torres SA, Pedroso DM (2010) Molecular dynamics simulations of complex-shaped particles using Voronoi-based spheropolyhedra. Physical Review E 81(6):061303

14. Hart R, Cundall PA, Lemos J (1988) Formulation of a three-dimensional distinct element model-Part II. Mechanical calculations for motion and interaction of a system composed of many polyhedral blocks. International Journal of Rock Mechanics and Mining Sciences 25(3):117-125

15. Hemingway E, O'Reilly OM (2018) Perspectives on Euler angle singularities, gimbal lock, and the orthogonality of applied forces and applied moments. Multibody System Dynamics pp 1-26

16. Henderson DM (1977) Euler angles, quaternions, and transformation matrices for space shuttle analysis. Tech. rep., NASA

17. Huang H, Tutumluer E (2014) Image-Aided Element Shape Generation Method in DiscreteElement Modeling for Railroad Ballast. Journal of Materials in Civil Engineering 26(3):527-535
18. Höhner D, Wirtz S, Kruggel-Emden H, Scherer V (2011) Comparison of the multi-sphere and polyhedral approach to simulate non-spherical particles within the discrete element method: Influence on temporal force evolution for multiple contacts. Powder Technol 208(3):643-656

19. Iaconeta I, Larese A, Rossi R, Oñate E (2017) An Implicit Material Point Method Applied to Granular Flows. Procedia Engineering 175:226-232

20. Indraratna B, Ngo NT, Rujikiatkamjorn C (2011) Behavior of geogrid-reinforced ballast under various levels of fouling. Geotextiles and Geomembranes $29(3): 313-322$

21. Indraratna B, Ngo NT, Rujikiatkamjorn C, Vinod JS (2014) Behavior of Fresh and Fouled Railway Ballast Subjected to Direct Shear Testing: Discrete Element Simulation. International Journal of Geomechanics 14(1):34-44

22. Irazábal J (2017) Numerical analysis of railway ballast behaviour using the Discrete Element Method. PhD thesis, URL https://www.scipedia.com/ public/Iraz\%E1bal_Gonz\%E1lez_0\%F1ate_2017a

23. Irazábal J, Salazar F, Oñate E (2017) Numerical modelling of granular materials with spherical discrete particles and the bounded rolling friction model. Application to railway ballast. Computers and Geotechnics 85:220-229

24. Kuipers JB (2002) Quaternions and Rotation Sequences: A Primer with Applications to Orbits, Aerospace, and Virtual Reality. Princeton University Press, Princeton, N.J

25. Liu Z (2011) Measuring the Angle of Repose of Granular Systems Using Hollow Cylinders. techreport, University of Pittsburgh

26. Lopes DS, Silva MT, Ambrósio JA, Flores P (2010) A mathematical framework for rigid contact detection between quadric and superquadric surfaces. Multibody System Dynamics 24(3):255-280

27. Luding S (2005) Molecular Dynamics Simulations of Granular Materials. In: The Physics of Granular Media, Wiley-Blackwell, pp 297-324

28. Munjiza A, Latham JP, John NWM (2003) 3d dynamics of discrete element systems comprising irregular discrete elements-integration solution for finite rotations in 3d. International Journal for $\mathrm{Nu}-$ merical Methods in Engineering 56(1):35-55

29. Nezami EG, Hashash YMA, Zhao D, Ghaboussi J (2006) Shortest link method for contact detection in discrete element method. International Journal for Numerical and Analytical Methods in Geomechanics 30(8):783-801

30. Oñate E, Idelsohn S, Del Pin F, Aubry R (2004) The particle finite element method. An overview. 
International Journal of Computational Methods 1(2):267-307, DOI 10.1142/S0219876204000204

31. Oñate E, Celigueta MA, Idelsohn SR, Salazar F, Suárez B (2011) Possibilities of the particle finite element method for fluid-soil-structure interaction problems. Computational Mechanics 48(3):307

32. Oñate E, Zárate F, Miquel J, Santasusana M, Celigueta MA, Arrufat F, Gandikota R, Valiullin K, Ring L (2015) A local constitutive model for the discrete element method. Application to geomaterials and concrete. Computational Particle Mechanics 2(2):139-160

33. O'Sullivan C, Bray JD (2004) Selecting a suitable time step for discrete element simulations that use the central difference time integration scheme. Engineering Computations 21(2/3/4):278-303

34. Podlozhnyuk A, Pirker S, Kloss C (2017) Efficient implementation of superquadric particles in Discrete Element Method within an opensource framework. Computational Particle Mechanics 4(1):101-118

35. Richefeu V, Mollon G, Daudon D, Villard P (2012) Dissipative contacts and realistic block shapes for modeling rock avalanches. Engineering Geology 149-150:78-92

36. Salazar F, Irazábal J, Larese A, Oñate E (2015) Numerical modelling of landslide-generated waves with the particle finite element method (PFEM) and a non-Newtonian flow model. International Journal for Numerical and Analytical Methods in Geomechanics 40(6):809-826

37. Santasusana M (2016) Numerical techniques for non-linear analysis of strutures combining Discrete Element and Finite Element Methods. PhD thesis

38. Santasusana M, Irazábal J, Oñate E, Carbonell JM (2016) The Double Hierarchy Method. A parallel $3 \mathrm{D}$ contact method for the interaction of spherical particles with rigid FE boundaries using the DEM. Computational Particle Mechanics 3:407-428

39. Taylor DW (1948) Fundamentals of soil mechanics. J. Wiley

40. Tian T, Su J, Zhan J, Geng S, Xu G, Liu X (2018) Discrete and continuum modeling of granular flow in silo discharge. Particuology 36:127-138

41. Whitmore SA (2000) Closed-form integrator for the quaternion (euler angle) kinematics equations

42. Zhang Y, Jia F, Zeng Y, Han Y, Xiao Y (2018) DEM study in the critical height of flow mechanism transition in a conical silo. Powder Technology 331:98-106

43. Zhao F, van Wachem BGM (2013) A novel Quaternion integration approach for describing the behaviour of non-spherical particles. Acta Mechanica
224(12):3091-3109

44. Zhou T, Hu B, Sun J, Liu Z (2013) Discrete Element Method Simulation of Railway Ballast Compactness During Tamping Process. The Open Electrical \& Electronic Engineering Journal 7(1):103109

45. Zhou W, Yang L, Ma G, Xu K, Lai Z, Chang X (2017) DEM modeling of shear bands in crushable and irregularly shaped granular materials. Granular Matter 19(2):25

46. Zhu HP, Zhou ZY, Yang RY, Yu AB (2008) Discrete particle simulation of particulate systems: A review of major applications and findings. Chemical Engineering Science 63(23):5728-5770 